Literatura y Lingüística $\mathrm{N}^{\circ} 22$

ISSN 0716-5811 / pp. 117-134

\title{
Tipos y funciones de las citas en artículos de investigación de tres disciplinas ${ }^{*}$
}

\author{
Omar Sabaj Meruane** \\ Dennis Páez Muñoz ${ }^{* * *}$
}

\section{Resumen}

En el marco del estudio de las características lingüísticas de los Artículos de Investigación a través de las disciplinas, este trabajo presenta una descripción de los tipos y funciones de las citas en un corpus de tres disciplinas: Lingüística, Filosofía y Terapia Psicológica. A partir de la aplicación de diferentes categorías (tipos y funciones), se analizaron textualmente las citas de 6 artículos. A pesar de la relativa proximidad de las disciplinas consideradas en el corpus, los resultados muestran una marcada variación entre ellas, tanto en los tipos como en las funciones de este tipo de textos. Estos resultados sugieren que las citas, como rasgo característico de los artículos de investigación, no tienen un uso ni una función homogénea en estos, y que esta variabilidad dice relación con la disciplina a la que pertenecen.

Palabras Clave: Tipos de citas - funciones de las citas - artículos de investigación

\begin{abstract}
Within the investigative framework of linguistic characteristics in Research Articles throughout the disciplines, this work presents a description of the types and functions of citations in a corpus comprised of three disciplines: linguistics, philosophy and therapeutic psychology. Using different categories (types and functions), citations from six articles were analyzed textually. Despite the relative proximity of the disciplines taken into consideration in the corpus, the results show a marked disciplinary variation, in types as well as functions, in this kind of text. These results suggest that citations as a characteristic feature of research articles do not have a homogeneous use nor function and that this variation indicates a relation with the discipline it pertains to.
\end{abstract}

Key words: Types of citation - citation functions - research articles

* Este trabajo se enmarca en el desarrollo del Proyecto FONDECYT 11080097: "El artículo de investigación a través de las disciplinas: el caso del indexador SCIELO Chile".

** Dr. en lingüística. Académico Universidad de La Serena, Prodicyt Centro de Investigación. omarsabaj@userena.cl

*** Licenciado en Educación. Profesor de Castellano y Filosofía. Universidad de La Serena. denpaez@hotmail.com 
Tipos y funciones de las citas en artículos de investigación de tres disciplinas / Omar Sabaj Meruane y Dennis Páez Muñoz

\section{Introducción}

Las citas son uno de los elementos lingüísticos más distintivos del discurso científico. Esto, debido a su función en la atribución del conocimiento, aspecto que le otorga a esta forma del uso del lenguaje la objetividad que de ella se espera. A pesar de su relevancia como rasgo característico del discurso científico, la descripción del uso de las citas en artículos de investigación en nuestra lengua es somera. Asimismo, la mayoría de los trabajos que se enfocan en la identificación de las citas en artículos de investigación, utilizan clasificaciones estrictamente textuales (directas e indirectas), sin una caracterización pormenorizada de las funciones específicas que cumplen estos tipos de textos en los géneros utilizados para la difusión del conocimiento científico. En este contexto, el propósito de esta investigación es describir los tipos y funciones de las citas en artículos de investigación de tres disciplinas. En el trabajo, se incorporan además otras clasificaciones textuales de las citas que, a pesar de su potencial interpretativo, no han sido utilizadas para nuestra lengua en la literatura revisada. El trabajo está organizado en las siguientes secciones. Se presenta, primero, una discusión teórica respecto de las citas, centrándonos en su clasificación desde el punto de vista textual y en las funciones específicas que estas cumplen en los artículos de investigación. Mostramos luego los pasos metodológicos seguidos en la investigación: los criterios de selección y justificación del corpus, los procedimientos analíticos y los cálculos estadísticos para la obtención de los resultados. A continuación, exponemos los principales hallazgos del trabajo, junto con una discusión de los mismos. Para finalizar, en la conclusión presentamos un breve resumen de la investigación, algunas reflexiones en torno a aspectos específicos y algunas de las líneas de proyección del estudio.

\section{Antecedentes}

\subsection{Las citas}

Las citas son un tipo de texto incrustado en otro texto. Reyes (1993) define las citas como la reproducción de otro discurso, un aspecto o una parte del mismo, en el propio. Para Romera (1996: 246) las citas son "la exposición que hace una persona de una idea expresada por un autor". Una definición más específica es la propuesta por Cázares, Christen, Jaramillo, Villaseñor y Zamudio (1980:81): "es la presentación, ya textual ya resumida, de aquellas ideas expresadas por otros autores que sirven de apoyo al investigador, se contraponen a lo que él dice o proporcionan mayor información sobre determinado tema". A partir de estas definiciones, se pueden establecer dos aspectos esenciales respecto de las citas: primero, su naturaleza textual (Reyes, 1993; Romera, 1996) y, segundo, su función (Cázares et al., 1980). Primero, respecto de su naturaleza, podemos especificar que la cita constituye un texto que, por definición, es dependiente de otro (forma ligada hipotáctica). Segundo, la cita establece un tipo específico

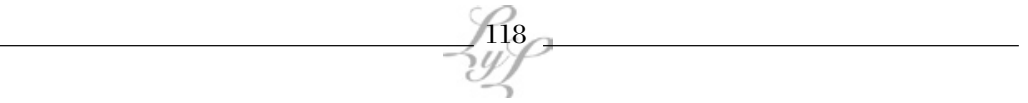


de relación (cumple una función) respecto del texto madre. Estas funciones, que detallamos en lo que sigue, se pueden definir y describir en muestras de textos reales para analizar su variación a través de distintos tipos de textos, en este caso, artículos de investigación científica.

\subsection{Tipos de citas}

En nuestro análisis, consideraremos dos clasificaciones de las citas. Una de ellas es de uso común y ha sido utilizada, con algunos matices, en otros estudios. A la segunda clasificación, por ser menos conocida, le dedicaremos una mayor extensión y detalle en su descripción.

\subsubsection{Citas literales (directas) y no literales (indirectas)}

La distinción entre citas literales y no literales es de amplio uso. Massi (2005), por ejemplo, propone una clasificación de los mecanismos de citación, distinguiendo citas en estilo directo e indirecto. Dentro del primer grupo, divide además el estilo directo en: antepuesto, el pospuesto y el discontinuo. Dentro del segundo grupo, subdivide las citas indirectas en los siguientes subtipos: 'indirecto genuino', 'indirecto con segmentos textuales' e 'indirecto narrativizado'. En este estudio no seguiremos directamente esta clasificación, pues su nivel de detalle no es acorde a los propósitos de este trabajo. Distinguiremos, en cambio, de forma genérica, las citas literales (directas) de las no literales (indirectas). En el primer caso, el autor incorpora las palabras verbatim de él mismo o, más comúnmente, de otro autor. En términos textuales, las citas literales suelen ir entre comillas (simples o dobles) y suelen, además del autor y el año, contener la referencia al número de página específico de la obra de la cual se extrajo. Las citas no literales, en contraste, suponen que el autor ha parafraseado, sintetizado o comentado las ideas de otro autor, por lo que no se reproducen las palabras tal como las utilizó el autor de la cita. En términos textuales, las citas no literales no contienen comillas y se pueden reconocer, pues aparece mencionado un autor y, en algunos casos, el año de la publicación.

\subsubsection{Citas Integrales y No Integrales}

Una distinción interesante que no ha sido aplicada en otros estudios revisados es la propuesta por Swales (1990), a saber, la diferencia entre Citas Integrales y No Integrales.

En el marco del Modelo Cars de la estructura retórica de las introducciones, se propone la existencia de Movidas Retóricas, entendidas como fragmentos textuales que dan cuenta de los propósitos comunicativos particulares que contribuyen a los propósitos globales de los textos. Estos propósitos se realizan a su vez en micropropósitos o "pasos". En el tercer paso, "revisión de la literatura previa" de la primera movida descrita para la estructura de las introducciones, a saber, "estableciendo el territorio"; Swales (1990) describe dos mecanismos 
Tipos y funciones de las citas en artículos de investigación de tres disciplinas / Omar Sabaj Meruane y Dennis Páez Muñoz

de citas que son los que nos ocupan en esta sección. Tal como el mismo Swales (1990) lo expresa, la ventaja de esta distinción es su fácil reconocimiento textual, pues se manifiesta en rasgos textuales superficiales: en las Citas Integrales, el autor de la cita cumple una función sintáctica en la oración en la que el autor del texto original hace la cita; en las citas no integrales, en cambio, el autor de la cita aparece, o bien entre paréntesis, o como una nota al pie de página, o al final del texto (Swales, 1990) y no establece una relación de dependencia con la oración del texto original. En las citas integrales, en cambio, el autor del texto original saca el nombre del autor del paréntesis y le otorga una función en la oración:

Figura 1: Ejemplo de Citas Integrales. Adaptado de Swales (1990: 149)

\begin{tabular}{|l|l|}
\hline Citas Integrales & Función sintáctica \\
\hline Swales (1990) muestra que... & Sujeto \\
\hline $\begin{array}{l}\text { La estructura de las introducciones ha sido descrita por } \\
\text { Swales (1990). }\end{array}$ & Agente \\
\hline Según Swales (1990),... & Adjunto reportativo \\
\hline
\end{tabular}

Figura 2: Ejemplo de Citas No-Integrales. Adaptado de Swales (1990: 149)

\begin{tabular}{|l|l|}
\hline Citas No-Integrales & Tipo \\
\hline $\begin{array}{l}\text { La descripción de la estructura de las introducciones (Swales, } \\
1990) \ldots\end{array}$ & En paréntesis \\
\hline $\begin{array}{l}\text { Diversas investigaciones se han centrado en la descripción de la } \\
\text { estructura de las introducciones } \ldots\end{array}$ & Con subíndice \\
\hline $\begin{array}{l}\text { Diversas investigaciones se han centrado en la descripción de la } \\
\text { estructura de las introducciones }{ }^{1} \ldots\end{array}$ & Con superíndice \\
\hline
\end{tabular}

En las figuras 1 y 2 , se presentan ejemplos de las Citas Integrales y de Citas No-Integrales. La importancia de esta distinción, en el marco de la propuesta de Swales (1990), es la relación que establecen estos mecanismos con pasos específicos asociados a su modelo de Movidas Retóricas, es decir, las funciones específicas que cumplen las citas en las distintas partes superestructurales de un artículo de investigación, aspecto que revisamos a continuación.

\subsection{La función de las citas en los Artículos de Investigación}

El estudio de las citas y la atribución del conocimiento, se ha consolidado como un objeto de estudio prolífero en el ámbito de la bibliometría (Siddharthan $\&$ Teufel, 2007; Urbano, 2001), sobre todo, en la determinación del factor de impacto de una revista (THOMSON REUTERS, 2010). A pesar de ello, la descripción de los tipos textuales de citas y, más aún, su función específica en artículos determinados, es mucho más escasa. Si bien existen modelos y listados de las funciones de las citas (Swales, 1990; Massi, 2005; Weinstock, 1981), los estudios en los que se aplican estos modelos o listados para la descripción de artículos, son excepcionales (Du Bois, 1988; Jacoby, 1987). En este apartado, revisaremos primero, de forma general, las funciones de las citas en la comuni-

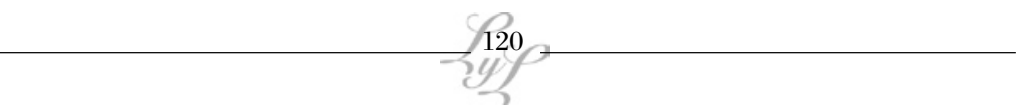


cación científica. Luego, describiremos los tipos funcionales de citas, y el detalle del modelo que utilizaremos en nuestro análisis.

Un primer aspecto que cabe mencionar, es el hecho de que el conocimiento científico se construye a partir de una red de relaciones entre distintas investigaciones o avances en un ámbito específico. En este contexto, las citas juegan un papel esencial en los artículos de investigación, pues tal como acertadamente señala Romera:

Es en este marco de la comunicación científica y de su función básica en la creación del conocimiento científico donde cobra sentido la exigencia de la práctica científica de que en la redacción de los trabajos de investigación se haga referencia explícita a aquellos trabajos científicos en los que se basan o apoyan las investigaciones realizadas. De este modo, las citas y referencias bibliográficas constituyen un elemento esencial de los trabajos de investigación y son una valiosa fuente de datos para el estudio de numerosas características del desarrollo científico. ( Romera, 1996: 245-246).

La autora (Romera, 1996) señala además otras funciones generales que cumplen las citas en la investigación, a saber:

a) Señalar el origen histórico del conocimiento.

b) Dar cuenta de la estructura social de la ciencia.

c) Indexar y recuperar información.

d) Establecer el estado de desarrollo de un ámbito.

e) Dar cuenta de la naturaleza colectiva de la construcción del conocimiento científico.

Coincidentemente, Massi (2005) señala otras funciones generales que cumplen las citas en la investigación. Según la autora, las citas se utilizan para:

a.1) Indicar y documentar la procedencia del conocimiento.

b.1) Recuperar los conceptos vertidos por la voz experta en el área que se investiga, por su relevancia y pertinencia.

c.1) Establecer relaciones entre un texto existente y el que está en elaboración, con la finalidad de comentar, asentir, disentir o señalar incongruencias.

Las funciones a y $\mathrm{d}$ son equiparables a las funciones a. 1 y b.1. Por su parte, la función c. 1 , se asocia a la función d.

Tanto Massi (2005) como Romera (1996), dejan entrever en sus postulados una idea que es definida y descrita en detalle por el interesante y novedoso trabajo de Greenberg (2009), a saber, el hecho de que en la elección de las citas por parte de un autor pueden generarse distorsiones de las ideas originales de un trabajo específico. A partir de un análisis, basado en la metodología de análisis de redes, Greenberg (2009) muestra cómo las citas pueden utilizarse 
Tipos y funciones de las citas en artículos de investigación de tres disciplinas / Omar Sabaj Meruane y Dennis Páez Muñoz

para establecer afirmaciones sin respaldo, garantía ni fundamento, lo que en definitiva demuestra que la inclusión de las citas, y la ciencia en general, distan mucho del afán objetivo y transparente que tradicionalmente se le ha asignado. Según Greenberg (2009), existen distintos tipos de citas que dan cuenta de distorsiones, sesgos, invenciones, falsas creencias, amplificación de las implicancias, entre otras.

Desde un punto de vista más específico, Swales (1990) destaca dos tipos funcionales, asociados a su modelo de Movidas Retóricas: las citas contrastivas (Jacoby, 1987) y las citas de síntesis o de resumen. En el primer caso, se trata de un tipo de cita en la que se va en contra de la conclusión que se está expresando en la oración misma. Textualmente, a menudo, estas citas contienen la abreviatura cfr. (conferatur, compárese o confróntese). Estas citas están relacionadas con los pasos 1 A, "indicar un contra argumento", o 1 B, "señalar un vacío", asociados a la Movida Retórica 2 de las introducciones, a saber, "estableciendo un nicho". En el segundo caso, el de las citas de síntesis o de resumen, se propone que su función sea la de hacer referencia general a un estado consensuado de conocimiento, o a un ámbito general de investigación. Estas citas, según Swales (1990), se vinculan con el paso "hacer generalizaciones respecto de un tema", constituyente de la Movida 1 de las introducciones, "estableciendo un territorio". En la figura 3, se presentan ejemplos de este tipo de citas:

Figura 3. Ejemplos inventados de citas contrastivas y de síntesis

\begin{tabular}{|l|l|}
\hline Citas contrastivas & Citas de síntesis \\
\hline Según los conductistas, la adquisición & La gramática generativa ha permitido \\
del lenguaje depende de los estímulos & dar nuevas luces sobre la adquisición \\
que recibe el niño (Cfr. Chomsky, & del lenguaje (Chomsky, 1957; Chomsky \\
1965). & 1965, Chomsky, 1981). \\
\hline
\end{tabular}

Para nuestro análisis de las citas en los artículos de investigación, utilizaremos la propuesta de Weinstock (1981). La ventaja de esta propuesta es su exhaustividad. El autor (Weinstock, 1981), propone un listado de 15 funciones que cumplen las citas en el discurso científico. Directa o indirectamente, la propuesta del autor comprende todas las otras funciones descritas en este apartado (Swales, 1990; Massi, 2005, Romera,1996 y, en menor medida, las descritas en el trabajo de Greenberg, 2009). A continuación, en la Figura 4, se presentan las funciones de las citas, según Weinstock (1981):

Figura 4. Las funciones de las citas en los artículos de investigación. Traducción propia de Weinstock (1981).

\begin{tabular}{|c|l|}
\hline $\mathbf{N}^{\mathbf{o}}$ en Weinstock (1981) $\mathbf{1}^{*}$ & Función \\
\hline 1 & Rendir homenaje a los pioneros \\
\hline 2 & Dar crédito a los trabajos relacionados \\
\hline 3 & Identificar metodologías, instrumentos, etc. \\
\hline 4 & Proporcionar trabajos de fundamentación \\
\hline 5 & Corregir los trabajos propios \\
\hline 6 & Corregir los trabajos de otros \\
\hline 7 & Criticar trabajos previos \\
\hline
\end{tabular}

122 


\begin{tabular}{|c|l|}
\hline 8 & Justificar afirmaciones \\
\hline 9 & Sugerir nuevas líneas de investigación \\
\hline 10 & $\begin{array}{l}\text { Proporcionar indicaciones de los trabajos de escasa } \\
\text { diseminación o indización }\end{array}$ \\
\hline 11 & $\begin{array}{l}\text { Refrendar datos, clases de hechos, constantes físicas, } \\
\text { etc. }\end{array}$ \\
\hline 12 & $\begin{array}{l}\text { Identificar las publicaciones originales en las que una } \\
\text { idea o concepto fue analizado }\end{array}$ \\
\hline 13 & $\begin{array}{l}\text { Identificar las publicaciones originales u otros trabajos } \\
\text { que describen un término o concepto eponímico }\end{array}$ \\
\hline 14 & Rechazar el trabajo o las ideas de otros \\
\hline 15 & Cuestionar pretensiones de prioridad de otros \\
\hline
\end{tabular}

* En lo que sigue, utilizaremos esta numeración original de Weinstock (1981)

\section{La investigación}

El objetivo de esta investigación es describir cuantitativa y cualitativamente la presencia de los tipos y funciones de las citas en un corpus de artículos de investigación.

\subsection{Corpus}

Debido a que este trabajo se inserta en un proyecto mayor, para la selección de nuestro corpus, se siguieron los criterios generales de inclusión y exclusión definidos en ese proyecto (Sabaj, Matsuda $\&$ Fuentes, 2010), a saber: que los artículos pertenecieran a alguna de las revistas indexadas en la Base Scielo-Chile, que su idioma original fuera el español, y que hayan sido publicados entre los años 2000 y 2008. Adicionalmente, en este trabajo decidimos enfocarnos en disciplinas humanistas y de las ciencias sociales, debido a que tal como hemos argumentado (Sabaj et al., 2010), tradicionalmente, estas disciplinas han sido mucho menos exploradas que otras. Luego de un análisis previo, las disciplinas incluidas fueron Lingüística (LIN), Filosofía (FIL) y Terapia Psicológica (TP). A partir de una base de datos general, utilizamos un sistema de generación de números aleatorios para seleccionar dos artículos por cada disciplina.

El corpus de análisis final, se muestra en la siguiente figura:

Figura 5. Conformación del corpus de análisis

\begin{tabular}{|c|c|c|c|c|c|}
\hline $\mathrm{N}$ & Código & Año & Disciplina & $\begin{array}{l}\text { Número de } \\
\text { palabras }\end{array}$ & $\begin{array}{c}\text { Número de palabras por } \\
\text { disciplina }\end{array}$ \\
\hline 1 & LIN 1 & 2008 & Lingüística & 5.824 & \multirow{2}{*}{14.473} \\
\hline 2 & LIN 2 & 2007 & Lingüística & 8.649 & \\
\hline 3 & FIL 1 & 2007 & Filosofía & 2.968 & \multirow{2}{*}{9.273} \\
\hline 4 & FIL 2 & 2006 & Filosofía & 6.305 & \\
\hline 5 & $\mathrm{TP1}$ & 2008 & Terapia Psicológica & 6.013 & \multirow{2}{*}{13.243} \\
\hline 6 & TP2 & 2007 & Terapia Psicológica & 7.230 & \\
\hline Total & & & & 36.989 & 36.989 \\
\hline
\end{tabular}

123 
Tipos y funciones de las citas en artículos de investigación de tres disciplinas / Omar Sabaj Meruane y Dennis Páez Muñoz

Para ver el detalle de los artículos analizados véase el Anexo 1.

\subsection{Categorías de análisis}

En la siguiente lista, se muestra una síntesis de las categorías incluidas en el análisis:

a) Tipos de citas

- Directas e indirectas (Masssi, 2005)

- Integrales y No-Integrales (Swales, 1990)

b) Funciones de las citas

- Funciones (Weinstock, 1981)

\subsection{Procedimientos de análisis}

Para el análisis de las citas se siguieron los pasos que se detallan a continuación. Para cada uno de los artículos se identificaron y se extrajeron las citas, y fueron traspasadas a una hoja de cálculo. Primero, se identificaron los tipos de citas (literales y no literales, Integrales y No-integrales) y se registraron los datos en la hoja de cálculos. Dado que esta clasificación depende de aspectos textuales fácilmente reconocibles (presencia de comillas, lugar del autor en el texto, etc.), el análisis fue expedito y sin mayores dificultades. Para la determinación de las funciones, los investigadores clasificaron de forma independiente las citas según las funciones de Weinstock (1981). Para ello se generó una lista de cotejo que se muestra en el Anexo 2. Como una forma de asegurar una clasificación correcta, se le pidió a dos pares expertos que realizaran el mismo ejercicio. De este análisis, se comprobó un alto acuerdo en las clasificaciones de los dos investigadores y de los pares expertos. En el caso de desacuerdo en la clasificación de la función, se optó por aquélla con mayor acuerdo entre los evaluadores.

Para la obtención de los resultados, se calculó la frecuencia absoluta; y para la comparación se calcularon porcentajes, respecto del conjunto de las citas totales de los artículos de cada una de las tres disciplinas. Además, se calculó un índice de citas por cantidad de palabras, dividiendo las citas por la cantidad de palabras de cada disciplina multiplicado por un factor común (1000). Así también, se estimó un índice de relación porcentual para asociar las dos categorías, que nos permitió saber cómo se distribuían porcentualmente las Citas integrales y No integrales, respecto de las citas directas e indirectas.

\section{Resultados y discusión}

En la Tabla 1, se presentan los datos generales respecto de la cantidad de citas identificadas por disciplina, su porcentaje respecto del total de citas analizadas y el índice de citas por cantidad de palabras. 
Tabla 1: Cantidad de citas por disciplinas, representación porcentual e índice de citas por número de palabras

\begin{tabular}{|c|c|c|c|}
\hline Disciplina & $\begin{array}{c}\text { Total de citas por } \\
\text { disciplinas }\end{array}$ & $\begin{array}{c}\text { \% de citas respecto del } \\
\text { total de citas }\end{array}$ & $\begin{array}{c}\text { Índice de citas por } \\
\text { cantidad de palabras }\end{array}$ \\
\hline LIN & 42 & 22 & 3 \\
\hline FIL & 77 & 41 & 8 \\
\hline TP & 69 & 37 & 5 \\
\hline Total & 188 & 100 & 5 \\
\hline
\end{tabular}

Tal como se aprecia en la Tabla 1, los artículos de Filosofía son los que, comparativamente, contienen más citas, seguidos por los de Terapia Psicológica y los de Lingüística. Concordantemente, se muestra que el índice de citas por cantidad de palabras sigue la misma tendencia. Tal como afirma Cañedo (1999), se podría sostener que la cantidad de citas de un artículo depende de su extensión. En este caso, sin embargo, esta idea se refuta. Al comparar estos resultados con los datos de la conformación del corpus (Figura 5), se puede observar que la cantidad de citas es inversamente proporcional al tamaño de los textos por disciplina. En efecto, los textos de Filosofía son más breves y proporcionalmente contienen más citas. Lo contrario de lo que ocurre en Lingüística. Estos datos sugieren que la cantidad de citas es un rasgo idiosincrático propio de la disciplina, aspecto que debería, sin embargo, comprobarse en una muestra ampliada de análisis.

A continuación, en el Gráfico 1, se muestra el porcentaje de citas directas e indirectas en cada disciplina:

Gráfico 1: Porcentaje de citas directas e indirectas

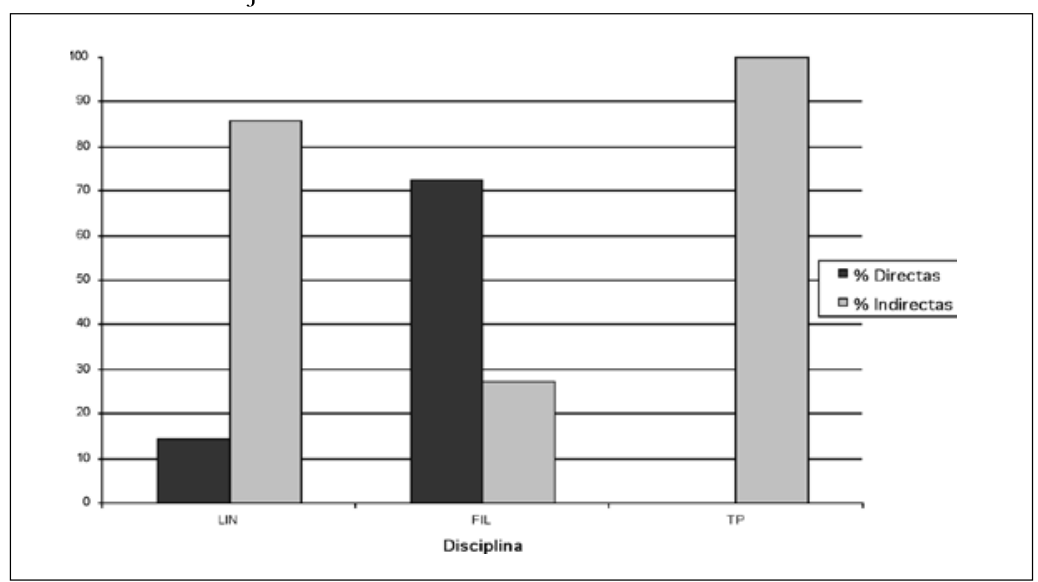

Tal como se muestra en el Gráfico 1, en Terapia Psicológica no se utilizan citas directas y su aparición es menor en Lingüística. En Filosofía, en cambio, el porcentaje de citas directas es notoriamente superior al de las indirectas. El mayor uso de citas indirectas en una disciplina da cuenta de la no necesidad de referir, en forma literal, a las palabras de otros autores en esa disciplina, por 
Tipos y funciones de las citas en artículos de investigación de tres disciplinas / Omar Sabaj Meruane y Dennis Páez Muñoz

lo que predomina el parafraseo, referencia general o síntesis de las ideas de otros autores. Este mayor uso de citas indirectas puede estar determinado por la existencia de un mayor conocimiento y acuerdo con las ideas que aportan los autores en cada disciplina, por lo que un análisis pormenorizado de las palabras que utiliza el autor para transmitir esas ideas se hace irrelevante. En Filosofía en cambio, la mayor aparición de citas directas revelaría una tendencia contraria. La naturaleza interpretativa y reflexiva de la Filosofía estaría determinando esa necesidad de reproducir las palabras tal cual como las expresó el autor citado, para así proporcionar un análisis detallado y pormenorizado de esas ideas. Al contrario de las otras disciplinas, el mayor uso de las citas directas en Filosofía, podría deberse a un menor grado de difusión y acuerdo de las ideas de los autores citados.

En el Gráfico 2, se expone el porcentaje de Citas Integrales y Citas No Integrales en cada una de las disciplinas:

Gráfico 2: Porcentaje de Citas Integrales y No Integrales

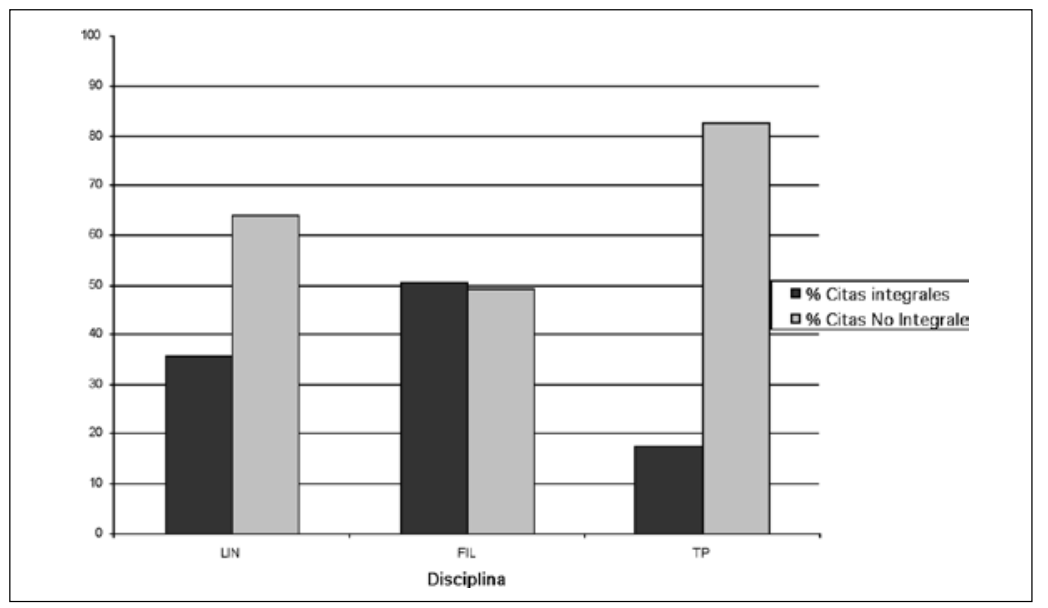

$\mathrm{Al}$ analizar el Gráfico 2, se puede observar que tanto en Lingüística como en Terapia Psicológica son más frecuentes las Citas No Integrales. En Filosofía, en cambio, la diferencia es mínima, y se opta indistintamente por Citas Integrales o No Integrales. Cuando el autor de un artículo de investigación utiliza una Cita Integral elige (consciente o inconscientemente) poner al autor de una idea en una posición de prominencia cognitiva respecto de la idea en sí misma, a saber, fuera del paréntesis como un elemento funcional de la oración. La necesidad de mencionar explícitamente al autor de la cita determina el tipo de función que el autor del artículo le otorga a esa cita (por ejemplo, si es una cita contrastiva o de síntesis). La explicitación del nombre del autor en una posición prominente es funcional, sobre todo, cuando el objetivo general del artículo principal es aclarar un concepto específico en un autor determinado, rasgo común en Filosofía (Léase FIL 1, en Anexo 1). Así entonces, este tipo 
de citas será más frecuente en aquellas disciplinas donde las ideas de autores específicos tienen un impacto determinante en esa disciplina. Por el contrario, en las disciplinas en las que existen programas de investigación consolidados, escuelas o líneas identificables, las Citas Integrales deberían disminuir. Por su parte, la mayor frecuencia de las Citas No integrales, en psicología y en Lingüística, serían coincidentes con una pretensión de objetividad cientificista a través de una despersonalización del discurso: el foco está más en las ideas y no en las personas puntuales que las propusieron.

Como una forma de analizar la relación entre ambos tipos de citas (directas e indirectas, por un lado, e Integrales y No Integrales, por otro), calculamos cómo se distribuían porcentualmente estos tipos, a saber, determinamos del total de citas directas e indirectas, cuántas son además Integrales y No Integrales.

En el Gráfico 3, se muestra esta relación:

Gráfico 3: Relación entre los dos tipos de citas

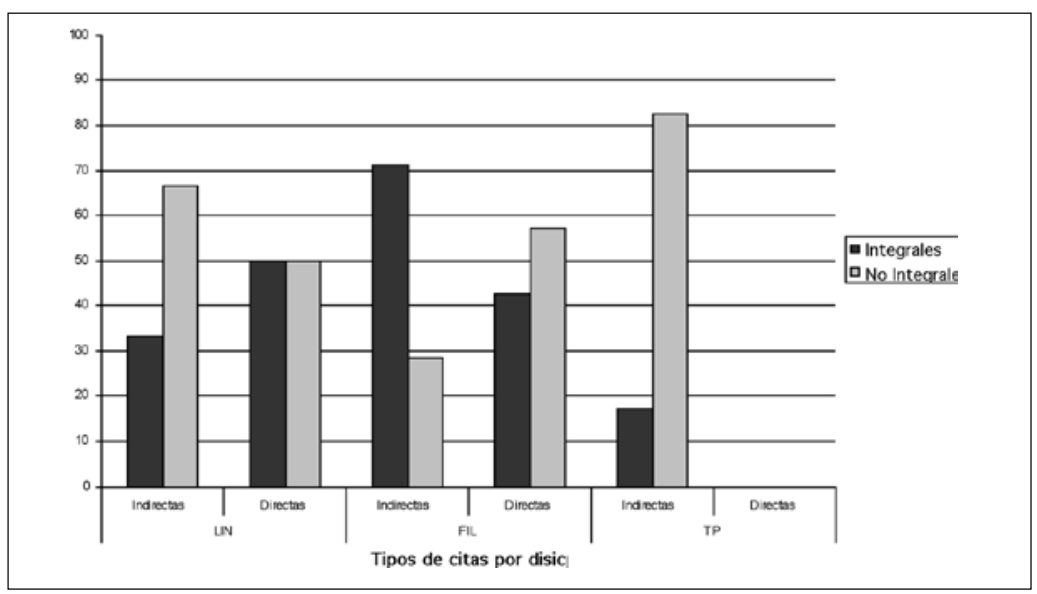

En estos datos, se observa nuevamente una tendencia común en Lingüística y en Terapia Psicológica. En los artículos de estas disciplinas, las citas indirectas tienden mayoritariamente a ser No integrales. En Filosofía, en cambio, las citas indirectas son mayoritariamente Integrales. El hecho de que las citas indirectas sean además No Integrales, implica que no es necesario reproducir las palabras tal cual como fueron formuladas por los autores de las citas y, además, que el autor es menos importante que la idea a la que se hace referencia. En los casos en que se utilizan las citas indirectas en Filosofía, se podría establecer que el autor sigue siendo más importante que la idea, por lo que, aunque no se reproduzcan las palabras literales de un autor, su figura se dispone en una posición de prominencia con respecto a la idea. Como hemos dicho, el uso de citas indirectas y No integrales supone una mayor objetivización del discurso $y$, por otra parte, determina el tipo de funciones que esa cita puede cumplir. En el caso de las citas directas, vemos que en Lingüística, pueden corresponder, 
Tipos y funciones de las citas en artículos de investigación de tres disciplinas / Omar Sabaj Meruane y Dennis Páez Muñoz

indistintamente, a Citas Integrales o No Integrales. En Filosofía, en cambio, las citas directas tienden, en su mayoría, a ser No Integrales. Es decir, se opta por reproducir las ideas literales de un autor pero ese autor queda en una posición menos relevante en el texto (entre paréntesis, en un subíndice o en un superíndice).

A continuación, nos enfocamos en las funciones de las citas en los artículos indagados. En primer lugar, cabe destacar que existe un conjunto de funciones que no aparecen en el corpus analizado. En la Tabla 2, aparecen estas funciones:

Tabla 2: Funciones no encontradas

\begin{tabular}{|c|l|}
\hline $\begin{array}{c}\text { No en Weinstock } \\
(1981)\end{array}$ & \multicolumn{1}{|c|}{ Función } \\
\hline 5 & Corregir los trabajos propios. \\
\hline 9 & Sugerir nuevas líneas de investigación. \\
\hline 10 & $\begin{array}{l}\text { Proporcionar indicaciones de los trabajos de escasa diseminación } \\
\text { o indización. }\end{array}$ \\
\hline 11 & Refrendar datos, clases de hechos, constantes físicas, etc. \\
\hline 13 & $\begin{array}{l}\text { Identificar las publicaciones originales u otros trabajos que } \\
\text { describen un término o concepto eponímico. }\end{array}$ \\
\hline 14 & Rechazar el trabajo o las ideas de otros. \\
\hline 15 & Cuestionar pretensiones de prioridad de otros. \\
\hline
\end{tabular}

Tal como lo han mostrado diversos estudios (ver punto 1.3), las disciplinas son sensibles a las funciones que se otorgan a las citas en sus artículos. Desde este punto de vista, la no ocurrencia de las funciones de la Tabla 2 implica que estas disciplinas no favorecen estos usos de las citas, y son un dato relevante para comprender la forma como se construye el conocimiento en estas disciplinas. Estas funciones tienen, entonces, un escaso potencial descriptivo al momento de analizar citas de las disciplinas acá consideradas.

Las funciones de las citas en los artículos analizados aparecen a continuación, en la Tabla 3:

Tabla 3: Porcentaje de aparición de las funciones de las citas en cada disciplina

\begin{tabular}{|c|l|c|c|c|}
\hline $\begin{array}{c}\mathrm{N}^{\circ} \text { en } \\
\text { Weinstock }\end{array}$ & \multicolumn{1}{|c|}{ Función } & $\%$ LIN & $\%$ FIL & $\%$ TP \\
\hline $\mathbf{1} 1$ & Rendir homenaje a los pioneros & 0 & 3 & 6 \\
\hline 2 & Dar crédito a los trabajos relacionados & 29 & 8 & 48 \\
\hline 3 & Identificar metodologías, instrumentos, etc. & 21 & 0 & 14 \\
\hline 4 & Proporcionar trabajos de fundamentación & 40 & 70 & 22 \\
\hline 6 & Corregir los trabajos de otros & 0 & 1 & 3 \\
\hline 7 & Criticar trabajos previos & 0 & 0 & 3 \\
\hline 8 & Justificar afirmaciones & 0 & 4 & 3 \\
\hline 12 & $\begin{array}{l}\text { Identificar las publicaciones originales en las } \\
\text { que una idea o concepto fue analizado }\end{array}$ & 10 & 14 & 1 \\
\hline Total & & 100 & 100 & 100 \\
\hline
\end{tabular}

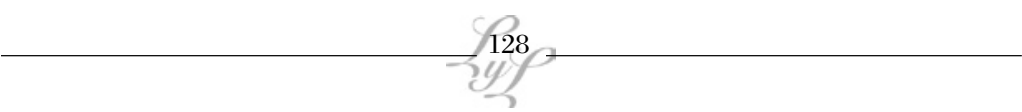


En la Tabla 3, surgen diversos datos interesantes de comentar o analizar. Para ello, analizaremos primero tres aspectos generales que dicen relación con la comunalidad, la especificidad y la variabilidad de funciones. Las funciones 2 , 4 y 12 son comunes a todas las disciplinas analizadas. Se podría establecer que, en la medida que una función es común a todas las disciplinas, estas funciones son poco sensibles a la diferencia epistemológica de las disciplinas y que, por lo tanto, estarían ligadas más bien a la naturaleza de la construcción del conocimiento a través de artículos de investigación. En otras palabras, se trataría de funciones básicas que deberían asomar en cualquier artículo de investigación, independiente de su disciplina. Así también, aparecen ciertas funciones específicas a una o a dos disciplinas. Por ejemplo, el caso de la función 7 que sólo ocurre en Filosofía, la función 1, 6 y 8 que es compartida por Filosofía y Terapia Psicológica. Por último, la función 3 sólo es compartida por Lingüística y por Terapia Psicológica y no aparece en Filosofía. Al contrario del caso anterior, en el que se expresan funciones comunes, la no aparición de una función específica en una disciplina nos proporciona información relevante de los supuestos epistemológicos de ella. Por ejemplo, dado que sabemos que la Filosofía es una disciplina reflexiva y eminentemente teórica, es esperable que la función número 3 no sea relevante, ya que es una función relacionada con disciplinas de corte más empírico, como la Lingüística y Terapia Psicológica. Por último, referente a estos aspectos generales, resulta pertinente señalar además que las disciplinas tienen un comportamiento diferenciado con respecto a la variabilidad de funciones que contienen: 8 TP > 6 FIL > 4 LIN. La mayor variabilidad de tipos funcionales de citas en una disciplina, no tiene una explicación directa. Más bien, se puede deber a factores diversos: la mayor producción de investigación en un ámbito, o a estilos o culturas disciplinares particulares.

En segundo y último lugar, también cabe comentar otros aspectos relevantes de los datos de la Tabla 3, enfocándonos en las funciones más prominentes de cada disciplina (marcadas en negrita en la Tabla 3). Tanto en Lingüística como en Filosofía, la función 4, 'proporcionar trabajos de fundamentación', es la más prominente. En Terapia Psicológica, en cambio, es la función 2, 'dar crédito a los trabajos relacionados', la más frecuente. Estos datos, creemos, se explicarían por una mayor afinidad o cercanía disciplinar entre la Lingüistica y la Filosofía, como humanidades, que entre ellas y el ámbito de la Terapia Psicológica, como una ciencia social. Esta diferencia también da cuenta de la naturaleza de cada disciplina. La necesidad de sustentar los argumentos que se presentan en los artículos para desarrollar conceptos (Filosofía y Lingüística) versus la necesidad de contextualizar el trabajo propio con otros trabajos similares (en Terapia Psicológica) estarían siendo la base de esta diferencia. En Terapia Psicológica, entonces, al ser la función 2 la más frecuente, lo que típicamente se realiza es una referencia a los objetivos, metodologías y resultados de un trabajo, de una manera integral y sintética. En forma esquemática, corresponde a: los autores $\mathrm{X}$ hicieron un estudio con sujetos de las características $Y$, llegando a los resultados Z. De este modo, el trabajo propio, en Terapia Psicológica, tendría además la 
Tipos y funciones de las citas en artículos de investigación de tres disciplinas / Omar Sabaj Meruane y Dennis Páez Muñoz

posibilidad de ser comparado con el trabajo citado en la sección de resultados o discusión.

\section{Conclusiones}

Las citas constituyen un rasgo esencial en la producción de la ciencia, específicamente cuando ésta se materializa en un artículo de investigación. En este trabajo, se indagaron los tipos y funciones de las citas en seis artículos de investigación de tres disciplinas. Tal como se observó, el comportamiento, tanto de los tipos como de las funciones que tienen las citas, es variable a través de las disciplinas. En primer término, se estableció que, comparativamente, los artículos de Filosofía contienen una mayor cantidad de citas que los artículos de las otras disciplinas. Además, desde un punto de vista general, tanto en Terapia Psicológica como en Lingüística las citas tienden a ser indirectas, mientras que en Filosofía suelen ser directas. Resulta significativo destacar la ausencia total de citas directas en Terapia Psicológica, lo que implicaría que en esta disciplina la reproducción literal de las palabras de otros autores no cumple una función relevante. Al analizar las Citas Integrales y No Integrales, constatamos que éstas son claramente más frecuentes que las primeras, tanto en Lingüística como en Terapia Psicológica.

Un aspecto interesante que se puede seguir explorando es la relación de cercanía o lejanía que se establece entre el autor de la cita y el autor del artículo, a partir de los tipos de citas que se utilizan en cada disciplina. De forma más o menos directa esta relación se podría esquematizar de la forma en la que se presenta en la siguiente figura:

Figura 6: Relación entre tipos de citas y cercanía o lejanía entre el autor de la cita y el autor del artículo

\begin{tabular}{|l|c|l|c|}
\hline \multicolumn{4}{|c|}{ Autor del artículo / Autor de la cita } \\
\hline + Cercanía & $\begin{array}{c}\text { Directas No } \\
\text { integrales }\end{array}$ & Indirectas Integrales & $\begin{array}{c}\text { Indirectas No } \\
\text { integrales }\end{array}$ \\
\hline Directas integrales
\end{tabular}

Con respecto a las funciones de las citas en los artículos analizados, es importante destacar que muchas de esas funciones no aparecen en el corpus analizado. Por otra parte, tal como lo describimos, existen algunas citas que son comunes a todas las disciplinas. Estas citas comunes se constituirían como transdisciplinares, es decir, no serían sensibles a ninguna disciplina en particular, y serían propias de la práctica general de escribir artículos de investigación. Al contrario, describimos casos en los que ciertos tipos de citas son específicas a una o dos disciplinas, y no aparecen en el resto. Esta ocurrencia específica daría cuenta de una afinidad entre esa función y los supuestos epistemológicos, metodológicos y los objetos de estudios de cada disciplina.

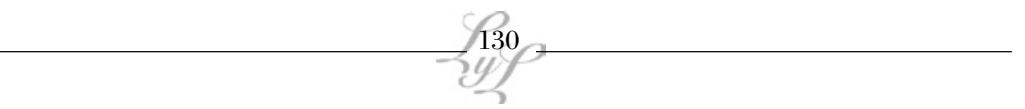


Es primordial acotar también que si bien las disciplinas acá consideradas son -desde un punto de vista general- bastante próximas, el uso de los tipos y las funciones de las citas en el corpus analizado es altamente variable.

A pesar de la imposibilidad de generalizar estos resultados, dados los procedimientos de recolección y tamaño del corpus, los hallazgos de esta investigación constituyen información relevante para cualquiera que se interese por los mecanismos de citas y la variación de los artículos de investigación a través de diferentes disciplinas.

Como proyección, esperamos poder replicar este estudio, incorporando artículos de otras disciplinas, específicamente, de las ciencias de la vida y de las ciencias exactas.

\section{Bibliografía}

Cañedo, R. (1999). "Los análisis de las citas en evaluación de los trabajos científicos y las publicaciones seriadas". ACIMED 7 (1), 30-39.

Cazáres, L.; Christen, M.; Jaramillo, E.; Villaseñor, L. \& Zamudio, L. (1980). Técnicas actuales de investigación documental. México: Trillas.

Du Bois, L. (1988). "Citation in biomedical Journal Articles". English for Specific Purposes 7 (3), 181-193.

Greenberg, S. (2009). "How citation distortions create unfounded authority: Analysis of a citation network" [en línea]. Disponible en: http://www. bmj.com/cgi/content/full/339/jul20_3/b2680

Jacoby, S. (1987). "References to other researchers in literary research articles". ELR Journal 1, 33-78.

Massi, M. (2005). "Las citas en la comunicación académica escrita" [en línea]. Disponible en: http://www.rieoei.org/deloslectores/1011Palmira.PDF

Reyes, G. (1993). Los procedimientos de citas: estilo directo y estilo indirecto: Madrid: Arco libros.

Romera, M. (1996). "Citas y referencias bibliográficas en el sistema de comunicación científica". Revista Complutense de Educación 7 (1), 243- 270.

Sabaj, O.; Matsuda, K. \& Fuentes, M. (2010). "Un modelo para la homogenización de las clases textuales de la Biblioteca Scielo Chile: La variabilidad del Artículo de Investigación a través de las disciplinas". Revista Información Tecnológica 21 (6), 133-148.

Siddharthan, A. \& Teufel, S. (2007). "Whose idea was this, and why does it matter? Attributing scientific work to citations" [en línea]. Disponible en: http://www.csd.abdn.ac.uk/ advaith/naacl07-final2.pdf

Swales, J. (1990). Genre analysis. English in academic and research settings. Cambridge: Cambridge University Press. 
Tipos y funciones de las citas en artículos de investigación de tres disciplinas / Omar Sabaj Meruane y Dennis Páez Muñoz

THOMSON REUTERS (2010). "The Thomson Reuters Impact Factor" [ en línea]. Disponible en: http://thomsonreuters.com/products_services/ science/free/essays/impact_factor/

Urbano, C. (2001). "El análisis de citas en trabajos de investigadores como método para el estudio del uso de información en bibliotecas". Anales de documentación 4, 243-266.

Weinstock, M. (1981). "Citation indexes". Encyclopedia of Library and Information Science 5, 16-40. New York: Marcel Dekker. 


\section{Anexo 1. Detalle de los textos analizados}

\section{Revista de Lingüística Teórica y Aplicada}

\section{LIN1}

Difabio, H. (2008). El test cloze en la evaluación de la comprensión del texto informativo de nivel universitario. Revista de Lingüistica Teórica y Aplicada 46 (1), 121-137.

\section{LIN2}

Coloma, C.; Peñaloza, C. \& Reyes, F. (2007). Producción de oraciones complejas en niños de 8 y 10 años. Revista de Lingüística Teórica y Aplicada 45 (1), 33-44.

\section{Revista de Filosofía}

\section{FIL 1}

Contreras, C. (2007). De lo oblicuo a lo aporético: responsabilidad, justicia y desconstrucción. Revista de Filosofía 63, 111-116.

\section{FIL 2}

Ralón, G. (2006). La dimensión arqueológica de la fenomenología. Revista de Filosofía 62, 5-17.

\section{Revista Terapia Psicológica}

\section{TP1}

Urzúa, A. (2008). Calidad de Vida y Factores Biopsicosociales en Patologías Médicas Crónicas. Revista Terapia Psicológica 26 (2), 207-214.

\section{TP2}

García, M. \& Ibañez, M. (2007). Apego e Hiperactividad: Un Estudio Exploratorio del Vínculo Madre-Hijo. Revista Terapia Psicológica 25 (2), 123-134. 
Tipos y funciones de las citas en artículos de investigación de tres disciplinas / Omar Sabaj Meruane y Dennis Páez Muñoz

\section{Anexo 2: Pauta de cotejo para la identificación de las funciones}

\begin{tabular}{|c|c|}
\hline Nombre de la cita & Criterio para su clasificación \\
\hline $\begin{array}{l}\text { 1. Rendir homenaje a los } \\
\text { pioneros }\end{array}$ & $\begin{array}{l}\text { ¿Se refiere al (los) autor(es) citado (s) con } \\
\text { expresiones positivas, elogiando su labor? }\end{array}$ \\
\hline $\begin{array}{l}\text { 2. Dar crédito a los trabajos } \\
\text { relacionados }\end{array}$ & $\begin{array}{l}\text { ¿La cita es validada por el autor como un trabajo } \\
\text { responsable y serio, en el cual se han demostrado } \\
\text { resultados? }\end{array}$ \\
\hline $\begin{array}{l}\text { 3. Identificar metodologías, } \\
\text { instrumentos, etc. }\end{array}$ & $\begin{array}{l}\text { ¿La cita va en función de identificar o señalar } \\
\text { metodologías, procedimientos o instrumentos } \\
\text { desarrollados anteriormente por otros } \\
\text { investigadores? }\end{array}$ \\
\hline $\begin{array}{l}\text { 4. Proporcionar trabajos de } \\
\text { fundamentación }\end{array}$ & $\begin{array}{l}\text { ¿La cita tiene relación con el tema central que } \\
\text { aborda el AI? ¿Son los extractos citados cimientos } \\
\text { para desarrollar la investigación? }\end{array}$ \\
\hline 5. Corregir los trabajos propios & $\begin{array}{l}\text { ¿El autor se refiere a publicaciones propias } \\
\text { anteriores, invalidando o reparando una postura o } \\
\text { punto de vista anterior? }\end{array}$ \\
\hline 6. Corregir los trabajos de otros & $\begin{array}{l}\text { ¿La cita es utilizada en función de oponerse a un } \\
\text { punto de vista de otro autor? }\end{array}$ \\
\hline 7. Criticar trabajos previos & $\begin{array}{l}\text { ¿La cita se refiere a una idea que tiene directa } \\
\text { relación con la superación de ideas anteriores de } \\
\text { otros autores o refutación de éstos? }\end{array}$ \\
\hline 8. 8. Justificar afirmaciones & $\begin{array}{l}\text { ¿La cita es utilizada con el fin de respaldar } \\
\text { afirmaciones realizadas por el autor del AI? ¿Avala } \\
\text { alguna tesis presentada por el autor del AI? }\end{array}$ \\
\hline $\begin{array}{l}\text { 9. Sugerir nuevas líneas de } \\
\text { investigación }\end{array}$ & $\begin{array}{l}\text { ¿La cita proporciona nuevas posibilidades de } \\
\text { indagación y exploración en el campo disciplinario } \\
\text { que aborda el AI? }\end{array}$ \\
\hline $\begin{array}{l}\text { 10. Proporcionar indicaciones } \\
\text { de los trabajos de escasa } \\
\text { diseminación o indización }\end{array}$ & $\begin{array}{l}\text { ¿La cita se refiere a autores poco difundidos o sin } \\
\text { mayor relevancia o consideración? }\end{array}$ \\
\hline $\begin{array}{l}\text { 11. Refrendar datos, clases de } \\
\text { hechos, constantes físicas, etc. }\end{array}$ & $\begin{array}{l}\text { ¿La cita es utilizada con el fin de refrendar datos de } \\
\text { otros autores? }\end{array}$ \\
\hline $\begin{array}{l}\text { 12. Identificar las publicaciones } \\
\text { originales en las que una idea o } \\
\text { concepto fue analizado }\end{array}$ & $\begin{array}{l}\text { ¿La cita es utilizada para especificar cuál es la fuente } \\
\text { original en la que se abordó un concepto o idea } \\
\text { incluido en el AI? }\end{array}$ \\
\hline $\begin{array}{l}\text { 13. Identificar las publicaciones } \\
\text { originales u otros trabajos } \\
\text { que describen un término o } \\
\text { concepto eponímico }\end{array}$ & $\begin{array}{l}\text { ¿La cita reconoce conceptos o términos elaborados } \\
\text { originariamente por autores determinados de un } \\
\text { área? ¿Se muestran a los creadores originales de un } \\
\text { concepto? }\end{array}$ \\
\hline $\begin{array}{l}\text { 14. Rechazar el trabajo o las } \\
\text { ideas de otros }\end{array}$ & $\begin{array}{l}\text { ¿La cita se muestra en oposición a otros puntos de } \\
\text { vista, negando la validez de las ideas planteadas por } \\
\text { otros autores? }\end{array}$ \\
\hline $\begin{array}{l}\text { 15. Cuestionar pretensiones de } \\
\text { prioridad de otros }\end{array}$ & $\begin{array}{l}\text { ¿La cita se utiliza para contrarrestar las ideas } \\
\text { predominantes en un área determinada, } \\
\text { cuestionando su primacía? }\end{array}$ \\
\hline
\end{tabular}

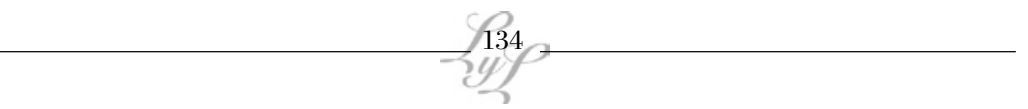

